\title{
Increasing charge lifetime in dc polarized electron guns by offsetting the anode
}

\author{
Omer Rahman, ${ }^{1}$ Erdong Wang, ${ }^{1, *}$ Ilan Ben-Zvi, ${ }^{1,2}$ Jyoti Biswas, ${ }^{2}$ and John Skaritka ${ }^{1}$ \\ ${ }^{1}$ Brookhaven National Laboratory, Upton, New York 11973, USA \\ ${ }^{2}$ Stony Brook University, Stony Brook, New York 11794, USA
}

(Received 7 May 2019; published 6 August 2019)

\begin{abstract}
Charge lifetime of strained superlattice Gallium Arsenide photocathodes in dc guns is limited by ion back bombardment. In this work, we propose and present simulation results for an offset anode scheme to increase charge lifetime in dc guns. In this scheme, the axial symmetry of the cathode-anode configuration is broken by offsetting the anode transversely, while keeping the laser spot on the electrostatic center. This eliminates the bombardment of high energy ions on the cathode and enables maximum usage of the available cathode area. Depending on the size of the available cathode area, this method can increase the charge lifetime by an order of magnitude compared to the current best alternative method. An anode assembly, capable of in-vacuum movement, is required for this method, which has been designed and fabricated at Brookhaven National Laboratory.
\end{abstract}

DOI: 10.1103/PhysRevAccelBeams.22.083401

\section{INTRODUCTION}

Strained superlattice GaAs photocathode based high voltage dc (HV-dc) guns have been used as sources of polarized electron beam in various facilities around the world [1-4]. These photocathodes can provide beam polarization up to $92 \%$, with quantum efficiency (QE) of about $1 \%$ at $780 \mathrm{~nm}$ laser illumination [5]. The photocathodes need to be coated with $\mathrm{Cs}$ and an oxidant $\left(\mathrm{O}_{2}\right.$ or $\mathrm{NF}_{3}$ ) to achieve "negative electron affinity (NEA)" on the surface. This NEA layer is highly sensitive to the vacuum levels of the gun and should be operated at extremely high vacuum. The degradation rate of cathode $\mathrm{QE}$ during operation determines how much charge can be extracted from a photocathode before it becomes unusable. The two widely used metrics to characterize HV-dc polarized guns are the fluence lifetime and the charge lifetime. Fluence lifetime is defined as the amount of charge extracted per unit area from a cathode before the QE drops to 1/e of its initial value [6]. Under constant operating conditions, fluence lifetime is a constant for a photogun and can be used to compare the performances of different guns [7]. Charge lifetime is defined as the total charge extracted from a photocathode until the quantum efficiency falls to 1/e of its initial value and can be used calculate the operation time

\footnotetext{
*wange@bnl.gov
}

Published by the American Physical Society under the terms of the Creative Commons Attribution 4.0 International license. Further distribution of this work must maintain attribution to the author(s) and the published article's title, journal citation, and DOI. of a gun [8]. If the operating conditions are fixed, the fluence lifetime of a gun can be used to estimate the charge lifetime for different laser sizes.

For HV-dc polarized electron guns, ion back bombardment (IBB) is the dominant lifetime-limiting process for cathodes during operation [9]. In this process, the electron beam ionizes the residual gas as it propagates through dc gap and downstream beam line. The ions that are generated downstream of the gap can be blocked by using a biased anode [10]. However, the ions generated in the dc gap cannot be blocked. These ions get accelerated toward the photocathode due to the dc electric field, eventually hitting the cathode surface. Operational experiences at several polarized electron beam facilities have shown a clear damage pattern corresponding to bombardment from ions [11].

The SLC gun has demonstrated extraction of 10-16 nC bunch charge at $\mu \mathrm{A}$ levels of average current [12]. Jefferson Lab group has demonstrated $4 \mathrm{~mA}$ average current operation at $1500 \mathrm{MHz}$ rep rate ( $\mathrm{pC}$ bunch charge) with $85 \mathrm{C}$ charge lifetime [13]. High bunch charge (nC level) with high average current (mA level) operation, with acceptable $\mathrm{kC}$ charge lifetime, is yet to be demonstrated and has been one of the main focuses of research in the field of polarized electron guns. Designing such a gun requires an iterative approach where multiple coupled parameters are considered based on the requirement from that particular gun. For example: if a polarized gun is required to deliver ultralow emittance beam, the laser spot must be small. This condition will set an upper limit on the average current for practical operation, since high current yields small charge lifetime and laser spot cannot be increased due to emittance 
restriction. On the other hand, for high charge lifetime from a high average current source, the laser spot will have to be larger. However, it cannot be too large to induce unacceptable emittance growth and downstream beam loss. Cathode size, anode aperture, beam line aperture etc. are among other parameters that will have to be optimized for this case as well. In this paper, we have focused on increasing charge lifetime with acceptable beam quality by using large laser spots and optimized the other parameters (total cathode size, anode aperture, beam pipe diameter etc.) accordingly.

Substantial improvement of charge lifetime, while extracting high current and high bunch charge, from a polarized electron source will be beneficial towards various future collider projects [14-16]. A high current polarized electron source can also be used in producing a high intensity polarized positron beam, which can be used in various collider applications, fixed target experiments, material science applications etc. [17].

Apart from improving the vacuum levels in a gun, multiple operational techniques have been explored to increase the charge lifetime of a polarized gun. These techniques include increasing the laser spot size, operating the laser off center, limiting the cathode active area and biasing the anode to block ions coming from downstream of the dc gap $[8,10,18]$. Offsetting the laser for higher charge lifetime has been used at MAMI Mainz and Jefferson lab, which is currently the standard mode of operation for polarized electron guns $[4,19]$. In this scheme, beam is extracted from a spot radially offset from the electrostatic center (EC) of the cathode. This ensures that the $\mathrm{QE}$ degradation under the laser spot is only due to low energy ion bombardment, since the higher energy ions get focused at the EC. The damage rate due to low energy ions is much slower compared to the damage due to higher energy ions and therefore this operation mode yields a higher charge lifetime [7]. One of the major limitations for this scheme is that it limits the maximum laser spot size to be much less than $50 \%$ of the available cathode area. Therefore, an opportunity of getting even higher charge lifetime by using a larger laser spot becomes impractical using offset laser scheme.

In this work, we propose an offset-anode scheme to completely eliminate high energy ion bombardment on the cathode surface to make maximum use of the available photocathode area. In this scheme, the axial symmetry of cathode-anode configuration is broken by transversely offsetting the anode, while keeping the laser spot on the center of the cathode. Depending on the size of the available photocathode area, the charge lifetime can be increased a factor of ten beyond the best alternate method.

This paper is arranged in three sections. Section II describes the offset-anode scheme, compares it with other schemes and provides a brief description of a gun that is being designed to test the scheme. Section III focuses on the ion back bombardment simulation for various offset-anode and offset-laser conditions. We demonstrate the separation of ion trajectories, compare the effectiveness of the two schemes in reducing ion back-bombardment damage and substantiate the claim for increased charge lifetime of the offset-anode scheme. Finally, Sec. IV deals with the feasibility of the scheme concerning electron beam dynamics in the offset-anode scheme. There we deal with the ability to transport the electron beam with no loss in the gun, and keeping a good emittance in this nontrivial 3-D transport problem.

\section{OFFSET ANODE SCHEME}

\section{A. Description of the scheme}

The energy range of the back bombarded ions play a crucial role in determining the damage rate for different operation schemes. For clarity, we will use the following convention to group back bombarded ions based on their energy: low energy ( $0-30 \%$ of maximum energy), medium energy (30\%-80\% of maximum energy) and high energy ( $80 \%-100 \%$ of maximum energy). It should be noted that the damage on the photocathode does not have clear thresholds in terms of ion energy and this convention is adopted for this particular paper to clarify the concepts and compare different operational schemes.

When the laser is operated at the center of the cathode, the entire laser illuminated area gets bombarded by ions with energy varying from low to high. The high energy ions at $80 \%$ to $100 \%$ of the maximum energy, are tightly concentrated at the EC due to the Pierce like focusing in the dc gap. The medium energy ions are not as concentrated as the high energy ions since they are generated closer to the cathode and beam size is larger than at the end of the dc gap. The low energy ions, generated within the first few $\mathrm{mm}$ from the cathode, are distributed over the entire laser illumination spot. Therefore, the entire laser illumination area is damaged to various degree depending on the energy of the back bombarded ions. This mode provides poor operating conditions and was shown to have poor fluence (charge) lifetime [7].

During offset-laser operation, the area under laser illumination avoids the high energy ion bombardment. It has been shown experimentally that the larger the offset, the higher the charge lifetime [7]. This experimental finding suggests that even though the laser spot is not bombarded by the high energy ions, medium and low energy ions still degrade cathode. As the offset amount increases, the laser illuminated area encounters less medium energy ions leading to a higher fluence (charge) lifetime. However, for the highest fluence lifetime in this scheme, the laser spot size is limited by two constraints: offset distance from EC and distance from the edge. The spot size has to be small enough such that it can fit within the electrostatic center and the edge of the photocathode. Beam extraction from the edge may result in extreme trajectory of the beam which 


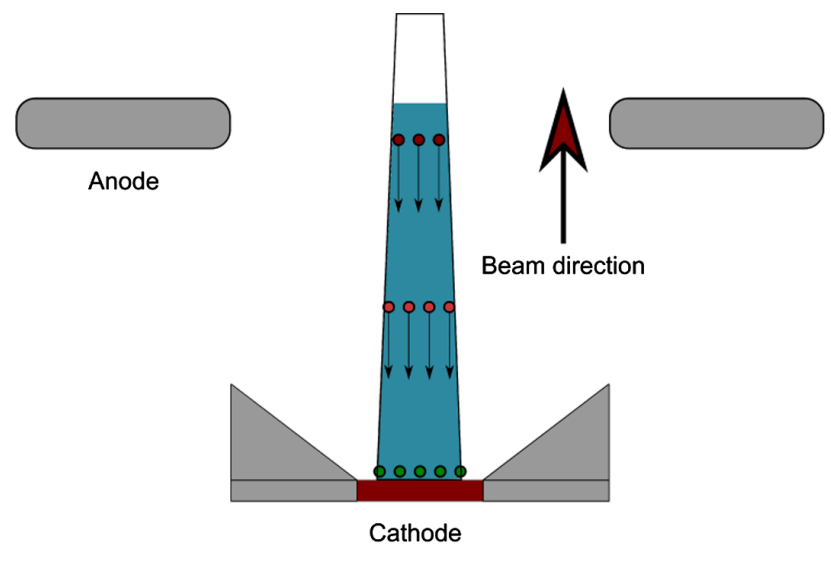

(a) On axis operation

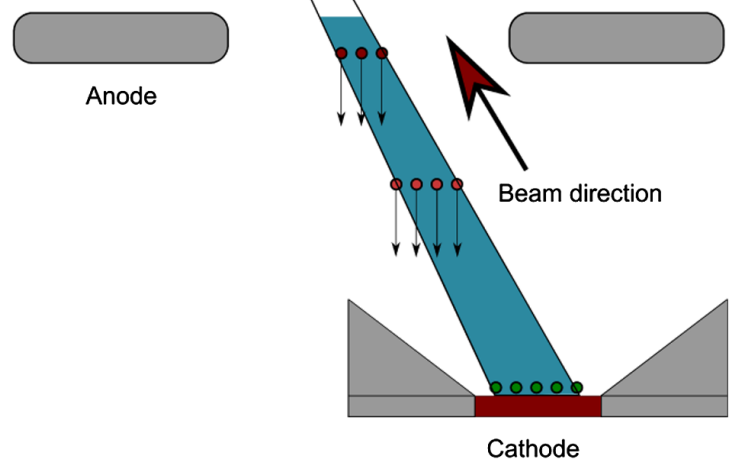

(b) Offset anode operation

FIG. 1. Ion generation at different points along the beam path in the dc gap for on-axis and offset-anode scheme. Green, light red and dark red circles indicate low, medium and high energy ions. The arrow on the ion circles indicates general direction towards which the ions tend to move. Effect of focusing on the medium and high energy ions are not shown.

may cause beam loss at the anode or nearby beam pipe. This will create unwanted outgassing and QE will degrade much faster than on-center operation [20]. These constraints restrict the maximum size of the laser spot to be less than $50 \%$ of the total available area of the photocathode. Grames et al. (2011) carried out extensive experimental studies to understand the dependency of charge lifetime on laser spot size and laser offset amount [7]. In their experiments, $3.5 \mathrm{kC}$ charge lifetime with $4 \mathrm{~mA}$ average current was achieved from a $1.5 \mathrm{~mm}$ laser spot, with $2 \mathrm{~mm}$ radial offset, from bulk GaAs at $532 \mathrm{~nm}$ laser operation. The diameter for this cathode was $12.8 \mathrm{~mm}$. Therefore, the majority of the available photocathode area remains unused. The laser spot can be moved from spot to spot on the photocathode, but that will require stopping the gun operation, possible reactivation of the cathode using a mask on the desired spot and finally tuning the beam optics for each new laser position.

To maximize the use of available photocathode area, while maintaining the highest fluence lifetime, we propose to offset the anode transversely and break the axial symmetry of the cathode-anode configuration. Unlike the offset-laser scheme, we propose to operate the laser on the center of the cathode. A transverse magnetic field, generated by a Helmholtz coil, can deflect the electron beam to the center of the offset anode at the exit of the gun. After that, a set of steering magnets can bring the beam back to the center of the beam pipe. The ions generated along the beam path will have different bombardment spots depending on the point of generation along the beam trajectory. The trajectory of the lower energy ions, generated close to the cathodes, will be similar compared to that of offset-laser scheme and they will bombard the area under the laser spot. However the medium and high energy ions, generated further down the beam path, will have different trajectories compared to that of the other schemes. These ions will be generated at locations transversely offset from the cathode and will completely miss the cathode given a sufficiently large offset. Figure 1 shows the possible ion generation at different points along the beam path for on-axis and offsetanode scheme. The transverse magnetic field in the dc gap will have minimal effect on the heavy hydrogen ions. These ions should follow a nearly straight trajectory from the point of creation to the cathode surface. So if the anode is offset transversely by $6 \mathrm{~mm}$, we should expect the high energy ions to hit the cathode area at about $6 \mathrm{~mm}$ off center. The anode could be offset to a suitable distance such that the medium and high energy ions completely miss the photocathode surface area. Figure 2 provides a visual representation of the laser spot position and energy profile



Laser on center

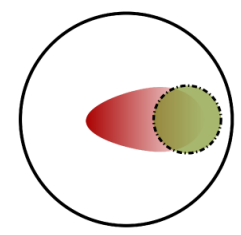

Laser off center

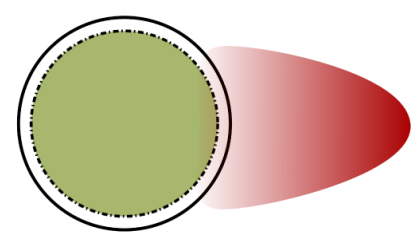

Anode off center

FIG. 2. Laser spot size and expected damage profiles for various operation schemes. The green area indicates damage from low energy ions and the red area indicates damage from medium-high energy ions. Increasing intensity of the red section represents increasing energy. The solid and dashed circles respectively indicate total cathode area and laser spot size. 
TABLE I. Comparison of maximum laser spot sizes for offsetlaser and offset-anode operation and expected charge lifetime increment factor.

\begin{tabular}{lccc}
\hline \hline $\begin{array}{l}\text { Cathode } \\
\text { radius } \\
\mathrm{mm}\end{array}$ & $\begin{array}{c}\text { Max. laser spot } \\
\text { (Offset-laser) } \\
\mathrm{mm}^{2}\end{array}$ & $\begin{array}{c}\text { Max. laser spot } \\
\text { (Offset-anode) } \\
\mathrm{mm}^{2}\end{array}$ & $\begin{array}{c}\text { Charge lifetime } \\
\text { increment } \\
\text { factor of }\end{array}$ \\
\hline 8 & 7.065 & 78.5 & 11.11 \\
9 & 12.56 & 113.04 & 9 \\
10 & 19.625 & 153.86 & 7.84 \\
11 & 28.26 & 200.96 & 7.11 \\
12 & 38.465 & 254.34 & 6.61 \\
13 & 50.24 & 314 & 6.25 \\
\hline \hline
\end{tabular}

of the back bombarded ions for on-axis, offset-laser and offset-anode schemes. Since the electrostatic center is now protected from medium-high energy ion bombardment, the cathode degradation will only be from low energy ions, similar to that of offset-laser scheme. However now that the laser spot can be operated at the electrostatic center, the laser spot size can be increased to maximize the usage of photocathode area. If ion back bombardment is the dominant mechanism of cathode degradation and there is no significant beam loss, the charge lifetime of a gun is directly proportional to the laser spot area. Therefore, offset-anode scheme provides a way to deliver beam with the largest possible laser size, while maintaining a constant fluence lifetime, resulting in the highest possible charge lifetime for the gun.

If $r$ is the radius of the available cathode, $a$ is the offset from the EC and $b$ is the clearance from the edge of the cathode, the maximum available area for laser spot in offset laser operation will be $\pi(r-a-b)^{2} / 4$. For offset anode operation, the available area for laser illumination will be $\pi(r-b)^{2}$. Experiments have shown that a $2-4 \mathrm{~mm}$ offset from the center and 3-4 mm clearance from the edge of the cathode maximizes the charge lifetime [7,21]. We can use these experimentally obtained parameters to estimate the improved charge lifetime resulting from the offset-anode scheme. The ratio of the areas can be used to estimate the increment of charge lifetime for offset anode scheme. As a conservative estimate, we use $a$ to be $2 \mathrm{~mm}$ and $b$ to be $3 \mathrm{~mm}$. Table I shows the estimated charge lifetime improvement stemming from the offset-anode scheme compared to the offset-laser scheme: the offset-anode scheme with larger laser spots results in factors of 6 to 11 improvement in charge lifetime.

We note that beam transport for loss-free beam delivery, including optimized design of cathode-anode assembly, is of prime importance for this scheme to be successful. However, the details of beam transport design are beyond the scope of this paper. In this particular paper, we will focus on proving the physics principle of the offset anode operation and its scope towards improving charge lifetime. In order to prove the feasibility of this scheme, we simulated and studied ion back bombardment in a dc gun for various laser and anode offset positions. The simulation results show that medium-high energy ions could indeed be shifted in an offset anode scheme. The required offset will depend on the total cathode area of the gun. The beam transport and emittance dilution for this scheme, for this particular gun, were also studied. A $5.2 \mathrm{nC}$ $1.2 \mathrm{~ns}$ bunch with $8.6 \mathrm{~mm}$ laser spot diameter could be transported out of the gun with a $13 \mathrm{~mm}$ anode offset without much degradation in beam quality.

\section{B. Description of the gun and simulation setup}

We used the BNL large cathode prototype gun and associated beam line for this simulation study [22]. This gun is designed for a $26 \mathrm{~mm}$ diameter cathode, with a nominal dc gap of $5.6 \mathrm{~cm}$. The maximum design voltage of this gun is $350 \mathrm{KV}$, providing $4 \mathrm{MV} / \mathrm{m}$ field on the center of the cathode. The aperture of the anode is $36 \mathrm{~mm}$ in diameter and the diameter of the beam line is $10 \mathrm{~cm}$. The anode section in the dc gun vessel has been modified to accommodate a recessed structure that enables mechanical movements of the anode using a special actuator assembly.

Figure 3 shows a CAD model and view of the anode mounting assembly during fabrication. The three actuators mounted on the middle plate on Fig. 3(b) are connected to a gear and chain arrangement outside vacuum. These three actuators provide $\mathrm{X}-\mathrm{Y}$ motion, pitch, and yaw and will be used for accurate alignment between cathode and anode. A separate actuator, attached to the side of the anode in Fig. 3(a), will be used to provide the necessary transverse offset. The leftmost circular plate [in Fig. 3(b)] is inside vacuum where the anode gets installed. The middle circular plate in Fig. 3(b) represents the point on the gun vessel where this assembly will be attached. The anode is electrically isolated and can be biased. The in-vacuum parts have been vacuum fired to achieve low outgassing rates.

Since the laser spot is at the center of the cathode, the beam will have to be deflected toward the center of the



(a) CAD model

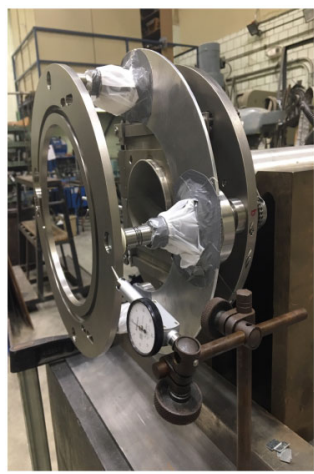

(b) During assembly
FIG. 3. CAD drawing and during fabrication pictures of the anode assembly. 


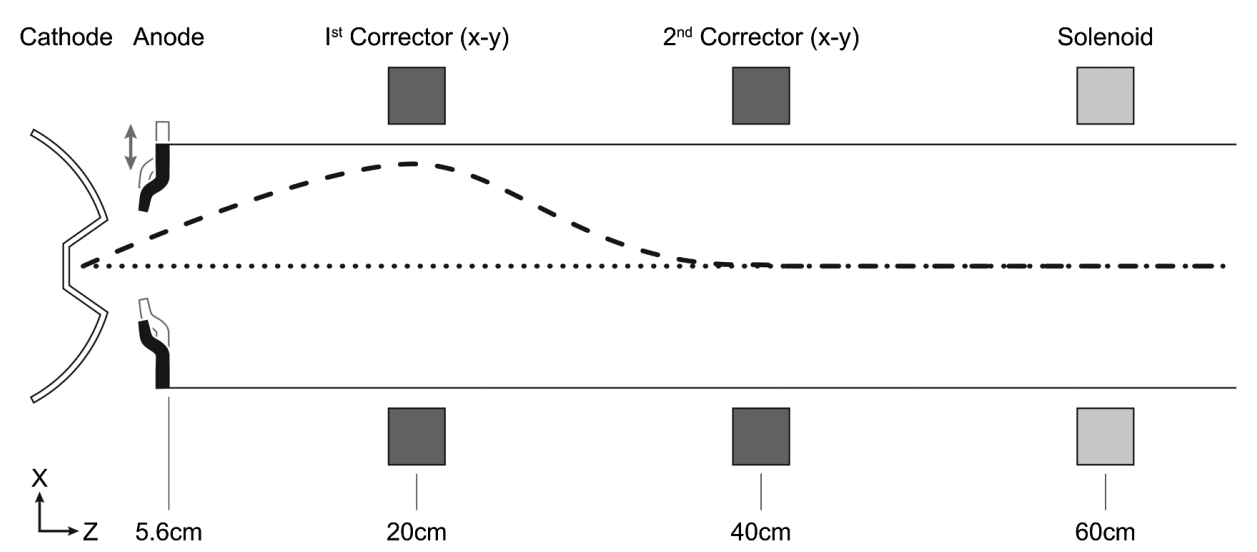

FIG. 4. Schematic of the beam line components used for simulation, the Helmholtz coil in the dc gap is not shown. The dotted and dashed lines represents on axis and offset anode operation, respectively. Components and distances are not to scale.

anode as it is exiting the dc gap. A Helmholtz coil, located outside of the gun vacuum chamber, can be used to deflect the beam to make it pass through the center of the anode. After the beam has passed the dc gap, subsequent steering magnets and solenoids are used to steer the beam on axis and compensate for emittance growth. The recessed structure and the chain assembly restrict how close the first X-Y steering magnets can be installed. For this design, $20 \mathrm{~cm}$ downstream from the cathode is the closest distance for the first steering magnet. The second set of steering magnets are at $40 \mathrm{~cm}$ and the solenoid is at $60 \mathrm{~cm}$ downstream. A schematic diagram of the beam line is shown in Fig. 4.

We used OPERA 3D to simulate the 3D electric field distribution in the dc gap for all different anode offset cases: on axis, $6 \mathrm{~mm}$ offset, $8.5 \mathrm{~mm}$ offset, and $13 \mathrm{~mm}$ offset [23]. All the displacements of the anode were in the positive X direction. The gradient at the center of the cathode is $4 \mathrm{MV} / \mathrm{m}$ and the $\mathrm{E}_{z}$ profile remains unchanged for various anode offsets as shown in Fig. 5. The longitudinal field


FIG. 5. Longitudinal $\left(\mathrm{E}_{z}\right)$ and transverse $\left(\mathrm{E}_{x}\right)$ electric fields along the beam path for various anode offsets. The plotted $\mathrm{E}_{x}$ field is what is experienced by the center of the beam as it is passes the dc gap. goes to zero at around $20 \mathrm{~cm}$ downstream from the cathode surface. Offsetting the anode introduces transverse focusing field, as shown in the bottom subplot of Fig. 5. The corrector magnets used in this simulation on the beam line are standard elements from "General Particle Tracer (GPT)" [24]. The solenoid field used in the simulation was the measured profile from the fabricated solenoid.

\section{ION BACK BOMBARDMENT: THEORY, SIMULATION RESULTS AND DISCUSSION}

\section{A. Theory of ion back bombardment}

Ion back bombardment rate, and consequent decay of cathode quality, is dependent on the vacuum parameters, current parameters, and voltage of the dc gun. At the beginning of the beam extraction, the entire laser spot area on the activated photocathode is capable of emitting electrons. As time progresses, the NEA layer deteriorates due to ion back bombardment and part of the laser illuminated area becomes incapable of electron emission. The decay rate of the emission area is directly proportional to the ion generation rate. If $A_{e}(0)$ is the emission area capable of emitting electrons at $t=0$ and $A_{e}(t)$ is the emission area at time $t$, the differential equation describing the decay of the emission area can be written as,

$$
\left(\frac{A_{e}(t)}{A_{e}(0)}\right)\left(\frac{d N_{i}}{d t}\right) S d t=-d A_{e}(t)
$$

where $\frac{d N_{i}}{d t}$ is the ion generation rate due to electron beam passing the dc gap and $S$ is the damage coefficient of the generated ions to the surface. The severity of cathode damage is dependent on the ion's energy. The higher energy ions will penetrate into the crystal and will result in lattice distortion and back sputtering while low energy ions may sputter off the surface atoms of activation layers [25]. Experimental studies have been performed to understand the surface layer damage after beam delivery but there is no conclusive quantitative model to describe the damage 
rate as the function of ion energy [26]. For simplicity, we neglect any dependence on ion energy that might exist as it relates to $\mathrm{QE}$ degradation (i.e., $S=1$ ).

The ion generation rate is directly proportional to the electron beam current, the ionization cross section, residual gas pressure and the electric field gradient. It can be written as,

$$
\frac{d N_{i}}{d t}=I \frac{n_{i}}{e} \int_{0}^{d} \sigma\left(E_{k}\right) d z=I \times F
$$

where $I$ is the electron beam current, $\sigma$ is the ionization cross section of hydrogen molecule, $E_{k}$ is the kinetic energy of the electron, $n_{i}$ is density of residual gas and $d$ is the dc gap distance. When the emission current is constant, the solution to Eq. (1) is of exponential form,

$$
A_{e}(t)=A_{e}(0) e^{\left(\frac{-t}{\tau}\right)}
$$

with the time constant, $\tau$, being related to available active area and incoming particle flux as,

$$
\tau=\frac{A_{e}(0)}{\left(\frac{d N_{i}}{d t}\right)}=\frac{A_{e}(0)}{I \times F} .
$$

Then Eq. (4) can be written as,

$$
A_{e}(t)=A_{e}(0) e^{-\kappa F t} .
$$

With $\kappa$ relating the average current and initial emission capable area as,

$$
\kappa=\frac{I}{A_{e}(0)} .
$$

From Eq. (4), it is evident that the time constant, hence the charge lifetime, will decrease if the average current is increased while keeping the initial emission area (equivalent of the laser spot size) fixed. In order to increase the charge lifetime for high average current, the laser spot size has to be increased accordingly. This is one of the major motivations behind the design of the BNL prototype gun, where the optimized laser spot size is determined to be $8.6 \mathrm{~mm}$ in diameter.

The ionization cross section can be written as [27],

$\sigma\left[m^{2}\right]=\frac{1.301 \times 10^{-24}}{\beta^{2}} f(\beta)\left[\ln \left(1.177 \times 10^{5} \beta^{2} \gamma^{2}\right)-\beta^{2}\right]$

with,

$$
f(\beta)=\frac{6.027 \times 10^{-5}}{\beta^{2}}\left(1.659 \times 10^{4} \beta^{2}-1\right) .
$$

With the electric field distribution along the beam path and using Eqs. (7) and (8), the ion generation flux, $F$, can be calculated.

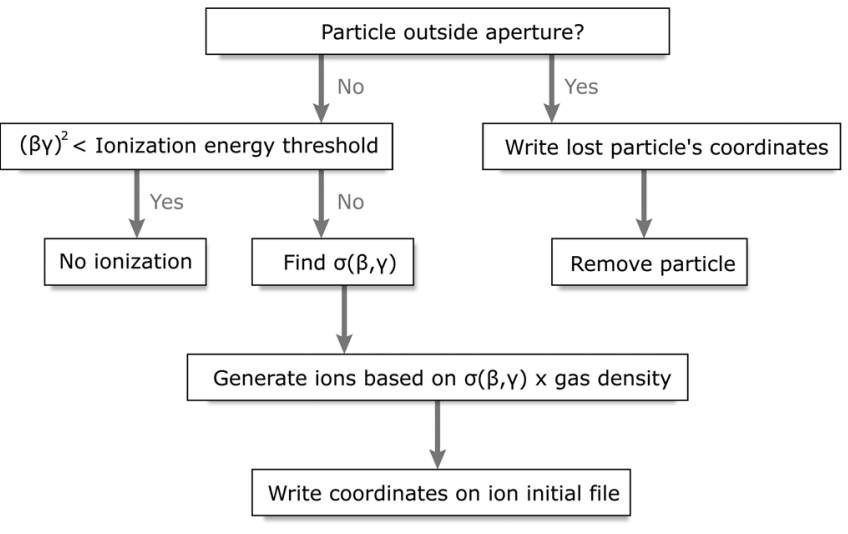

FIG. 6. Flow chart describing the ion generation algorithm used in this study.

We used GPT code to simulate both the electron beam and ion trajectories. A custom GPT element was written to simulate the ion generation along the path of the beam. The source code for the element can be found at the URL in Ref. [28]. Using Eq. (7) and (8), the ionization cross section for each electron at every time step can be calculated as the electrons are passing the dc gap. The ion generation rate, which is linear with the product of cross section and gas density, can then be calculated using Eq. (2). Figure 6 shows the flow chart for the ion generation algorithm.

The simulation was performed in two steps. First we simulated the electron beam, using the 3D electrostatic field profile obtained from OPERA 3D. The ions were also generated during this step, recording their initial positions as an initial ion distribution file. The initial hydrogen ion momentum distribution is a Maxwell distribution, where the velocities of the ions are thermal velocities and are negligible. Thus we assumed that the initial momentum of the generated ions to be zero. In the second step, the ions were accelerated using the initial ion distribution file in the same dc field. Since the trajectory of the electrons and ions are simulated separately, the effect of ion trapping in electron beam downstream of the anode was not considered [29]. In the dc gap, the trajectories of the electrons and ions for offset anode operations do not overlap entirely and therefore ion trapping in the dc gap can also be ignored. The leakage field through the anode aperture was considered and ions were generated up to approximately $20 \mathrm{~cm}$ downstream where the dc field goes to zero.

\section{B. Ion back bombardment simulation results}

The ion maps on the cathode surface for various anode and laser offsets are shown in Fig. 7, with the color bar representing the energy of the ions. The blue circle in Fig. 7 represents the laser spot, whereas the dashed black circle represents the total cathode area. The ionization cross section of hydrogen due to electron- $\mathrm{H}_{2}$ scattering is energy dependent and about 3 orders of magnitude higher for 


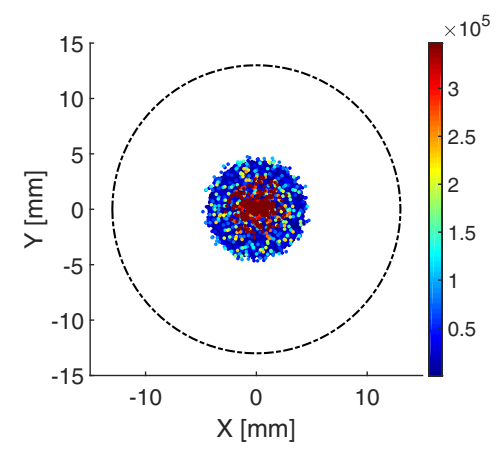

(a) On axis

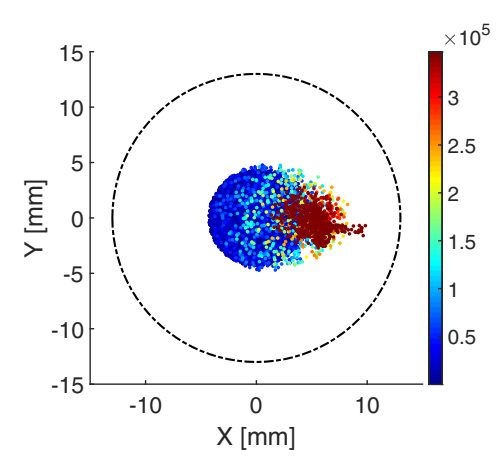

(d) Anode offset $6 \mathrm{~mm}$

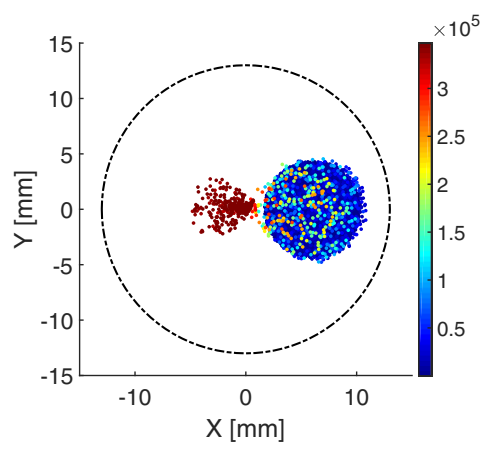

(b) Laser offset $6 \mathrm{~mm}$

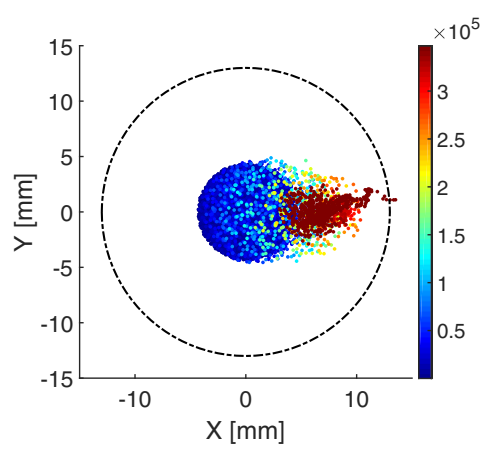

(e) Anode offset $8.5 \mathrm{~mm}$

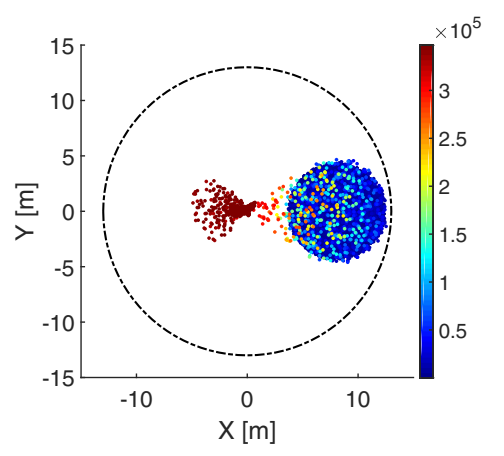

(c) Laser offset $8.5 \mathrm{~mm}$

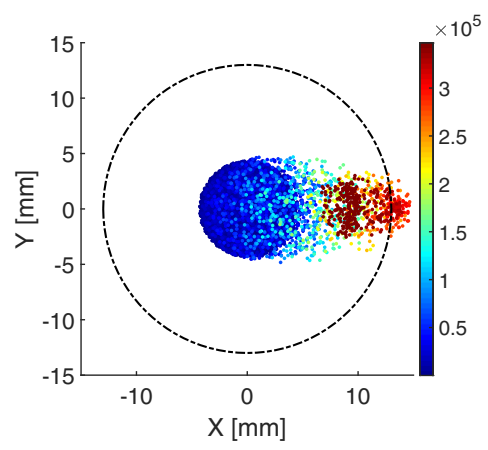

(f) Anode offset $13 \mathrm{~mm}$

FIG. 7. Simulated ion distribution on the cathode. Available cathode diameter is $26 \mathrm{~mm}$, represented by the dotted black circle. Laser spot is $8.6 \mathrm{~mm}$ in diameter, represented by the blue circle. The color bar represents the energy of the bombarding ions in eV. Electrostatic center for the cathode is at coordinate $(0,0)$.

lower (less than $100 \mathrm{eV}$ ) energy compared to higher energy (more than $100 \mathrm{keV}$ ) [27]. Therefore most of the ions generated in the dc gap are very low energy ions from within the first few $\mathrm{mm}$ of the cathode surface. The ion maps from Fig. 7 represents this with the high concentration of blue ions. For on-axis operation, Fig. 7(a), high energy ions are concentrated at the electrostatic center. This effect has been observed at Jefferson Lab [4]. Medium energy ions, represented by yellow and orange dots, are not as concentrated as the high energy ions and are seen to be present as far as the edge of the laser spot.

Figures 7(b) and 7(c) shows the ion map on the cathode for $6 \mathrm{~mm}$ and $8.5 \mathrm{~mm}$ laser offsets, respectively. In both cases, the higher energy ions are tightly concentrated at the electrostatic center. Some high energy ions are seen to be shifted in the opposite direction of the laser spot offset. These ions are generated when the beam is off axis on the dc gap-beam line and then get deflected in the dc gap due to the electric field from Pierce-like focusing. The further the radial distance of the ion from the central axis, the more deflection it will experience in the dc gap. The fact that the electrostatic center is heavily bombarded by high energy ions for any amount of laser offset restricts the maximum size of the laser spot. One important feature from two offset laser ion maps is the area covered by the medium energy ions. In Fig. 7(b), the medium energy ions (yellow and orange dots) cover about $70 \%$ of the laser spot, whereas in Fig. 7(c) they cover about $50 \%$ of the laser spot. This shows that with increasing laser offset, medium energy ion bombardment on the beam extraction spot decreases. This should result in increasing charge lifetime with increasing laser offset for a fixed laser spot size. This effect was also experimentally observed at Jefferson Lab [7].

For offset anode cases, the high energy ions are shifted towards the direction of the anode offset. With increasing anode offset, as seen in Figs. 7(d)-7(f), the higher energy ions are seen to have moved outside the laser spot on the cathode. The shift distance for high energy ions is equivalent of the anode offset distance. We notice by comparing all the offset anode cases is that the higher energy ions get more and more dispersed at the cathode with increasing anode offset. A closer inspection of the ion map for the $13 \mathrm{~mm}$ offset reveals that $350 \mathrm{keV}$ ions (dark red) are more dispersed than ions with energy between $300-350 \mathrm{keV}$ (light red). This is because these downstream ions are generated off center of the beam axis, on the positive quadrant of the XY plane. The $\mathrm{E}_{x}$ field experienced by these ions, as they are traveling to the cathode, is negative. The further off axis the ion is, the higher the magnitude of the $\mathrm{E}_{x}$ field, which results in the ions getting deflected by the time they reach the cathode. These ions could be eliminated by simply biasing the anode. The ions that are 


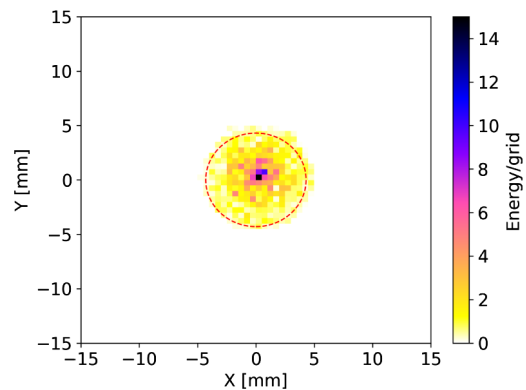

(a) On axis



(d) Anode offset $6 \mathrm{~mm}$



(b) Laser offset $6 \mathrm{~mm}$



(e) Anode offset $8.5 \mathrm{~mm}$

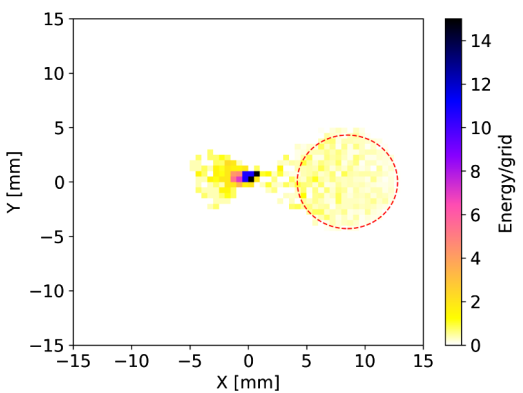

(c) Laser offset $8.5 \mathrm{~mm}$



(f) Anode offset $13 \mathrm{~mm}$

FIG. 8. Power deposited on the cathode due to ion back bombardment for various anode and laser offsets. The power deposition is calculated on $0.5 \mathrm{~mm}$ X $0.5 \mathrm{~mm}$ grids and normalized to the maximum power deposited in any grid. The red dotted circle represents the laser spot. Electrostatic center for the cathode is at $(0,0)$. The color bar represents the normalized power deposited, white being no power and black being maximum power.

generated close to the anode, but are in the dc gap, are not affected by this effect as much. These ions follow a straighter trajectory and hit the cathode at a distance almost equal to anode offset. Comparing all the offset-laser and offset-anode ion distributions from Fig. 7, it is clear that the laser size is limited in offset-laser scheme. For the offsetanode scheme, with suitable anode offset, the laser size could be increased to use most of the available photocathode.

The power deposited on the cathode was calculated on $0.5 \mathrm{~mm}$ by $0.5 \mathrm{~mm}$ grids using the energies of the bombarding ions for all cases shown in Fig. 7. The power per grid was normalized to the maximum power deposited on any grid and shown in Fig. 8. For on-axis and offsetlaser operation, $90 \%$ of the total power is deposited within a $3-5 \mathrm{~mm}^{2}$ area at the center of the cathode. For offset anode operation, the higher energy ions are distributed and shifted from the electrostatic center. For $8.5 \mathrm{~mm}$ and $6 \mathrm{~mm}$ anode offset, part of the laser spot is still affected by the higher energy ions. For $13 \mathrm{~mm}$ anode offset, the medium-high energy ions completely miss the laser spot at the center of the cathode.

The results in Table I were calculated assuming same fluence lifetime for offset-laser and offset-anode schemes, and that offset-anode scheme will protect the electrostatic center. Figures 7 and 8 show that it is indeed the case: with sufficient offset of the anode, the laser spot experiences similar ion energy and deposited power compared to offset-laser, while keeping the electrostatic center protected. Table II shows the normalized ion counts, under the laser spot, for all offset-laser and offset-anode cases for medium and medium + high energy ions. The ion counts for each case were normalized with respect to the count from on-axis operation. This comparison shows that offsetanode scheme is highly effective, even better than offsetlaser scheme, in reducing medium-high energy ion bombardment on the laser spot. For example: a $8.5 \mathrm{~mm}$ anode offset yields $36 \%$ less medium energy ions compared to $8.5 \mathrm{~mm}$ laser offset. This indicates that for the same offset amount, the offset-anode scheme should yield better fluence lifetime compared to the offset-laser scheme. Even though this improvement cannot be quantified directly, it indicates that charge lifetime improvement factors from Table I are

TABLE II. Normalized ion counts under the laser spot for all offset laser and anode cases. Ion counts for each case and energy range was normalized to that of the on-axis case.

\begin{tabular}{lccc}
\hline \hline Scheme & $\begin{array}{c}\text { Offset } \\
\text { amount } \\
(\mathrm{mm})\end{array}$ & $\begin{array}{c}\text { Normalized medium } \\
\text { energy ion } \\
\text { count }\end{array}$ & $\begin{array}{c}\text { Normalized } \\
\text { medium + high } \\
\text { energy ion count }\end{array}$ \\
\hline Offset-laser & $6 \mathrm{~mm}$ & 0.96 & 0.48 \\
Offset-laser & $8.5 \mathrm{~mm}$ & 0.75 & 0.35 \\
Offset-anode & $6 \mathrm{~mm}$ & 0.7 & 0.53 \\
Offset-anode & $8.5 \mathrm{~mm}$ & 0.48 & 0.27 \\
Offset-anode & $13 \mathrm{~mm}$ & 0.25 & 0.11 \\
\hline \hline
\end{tabular}


conservative estimates since it assumes same fluence lifetime for both schemes.

In reality, the vacuum in the dc gap is not uniform along the path of the beam and pressure profile could vary between the cathode surface to anode. To simplify the simulation, we only considered constant pressure profile in this simulation. However, the custom ionizer element can handle variable pressure profile as well.

\section{BEAM DYNAMICS SIMULATION}

The beam parameters used for this study are the nominal parameters for the proposed BNL large cathode inverted gun. A $5.3 \mathrm{nC}$ bunch with $1.2 \mathrm{~ns}$ bunch length and uniform density was simulated and propagated through the $2 \mathrm{~m}$ long beam line. The laser spot size is $8.6 \mathrm{~mm}$ in diameter, which is optimized for this particular gun design considering both cathode lifetime and beam quality. First we compare the beam quality for offset laser operation and offset anode operation. Then beam dynamics results for various offset anode operations are discussed. The beam dynamics study for offset anode operation focused on the trajectory of the beam (in particular the $\mathrm{X}$ trajectory, which is the axis of anode displacement), possible beam loss at various high risk spots on the beam line and emittance growth due to the transverse kick in the dc gap.

For the given laser spot size and overall cathode size in this gun, the maximum laser offset could be $8.5 \mathrm{~mm}$. Figure 9 compares the normalized $\mathrm{r}$ rms emittances $\left(\epsilon_{r}^{*}\right)$ for all laser and anode offset cases after compensation by the solenoid. For the $8.5 \mathrm{~mm}$ offset-laser case, the emittance is found to be $9.68 \mathrm{~mm}$-mrad, compared to $7.95 \mathrm{~mm}$-mrad for $13 \mathrm{~mm}$ offset-anode case. With increasing anode offset, the emittance seems to increase marginally which is expected. The $6 \mathrm{~mm}$ laser offset case emittance is almost equal to on axis operation case since this gun was specifically designed to tolerate that much laser offset. For $8.5 \mathrm{~mm}$ laser offset, the beam is almost approaching the edge of the cathode. This introduces nonlinearities due to external field which dominated the emittance growth in this case. This was

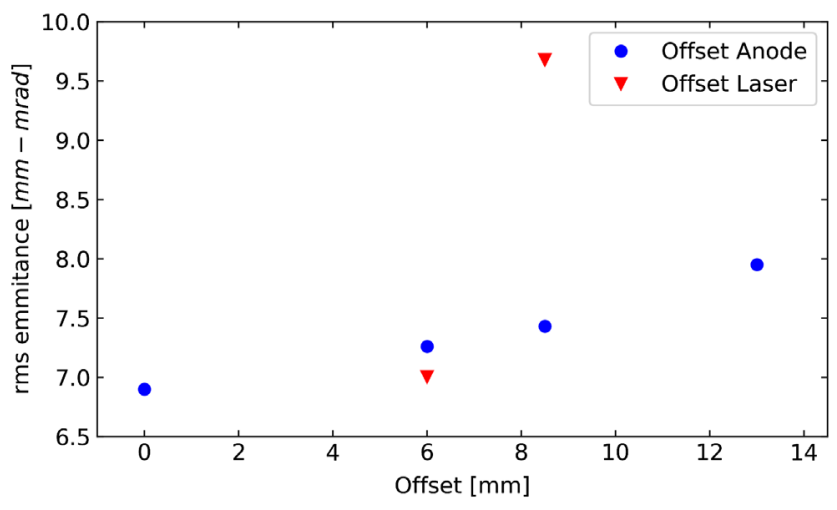

FIG. 9. Normalized $\mathrm{r}$ rms emittances $\left(\epsilon_{r}^{*}\right)$ for all offset anode and laser cases after compensation by the solenoid.
TABLE III. Ratios of normalized $x$ and $y$ emittances for all offset anode and laser cases.

\begin{tabular}{lcc}
\hline \hline Scheme & $\begin{array}{c}\text { Offset amount } \\
(\mathrm{mm})\end{array}$ & $\begin{array}{c}\text { Ratio of } \epsilon_{x}^{*} \\
\text { and } \epsilon_{y}^{*}\end{array}$ \\
\hline Offset-anode & 6 & 0.997 \\
Offset-anode & 8.5 & 0.976 \\
Offset-anode & 13 & 0.977 \\
Offset-laser & 6 & 0.98 \\
Offset-laser & 8.5 & 1.03 \\
\hline \hline
\end{tabular}

verified by comparing the emittance growth with and without space charge for various cases. This emittance growth due to nonlinear external field could not be compensated using a solenoid. The beam kinetic energy is $350 \mathrm{keV}$ where the space charge is still large resulting in sliced bunch rotation in transverse phase space. Therefore, we use normalized emittance $x, y$ at space charge compensated point as the parameter to characterize the beam astigmatism. We compared the ratios of $x-y$ normalized emittances after compensation. The results are tabulated in Table III. For all cases, the variation between $x$ and $y$ emittances are within 5\% which indicates acceptable beam quality.

Emittance growth due to geometric error or dc field aberration in the dc gun, for a small $0.35 \mathrm{~mm}$ laser spot, has been explored in Ref. [30]. In the presence of linear focusing and space charge, the transverse momentum $\mathrm{P}_{x}$ can be fitted at the exit of the gun as,

$$
P_{x}=C x+\alpha_{1} x^{3},
$$

where $C$ is the focusing constant and $\alpha_{1}$ is the aberration constant. The normalized transverse emittance is defined as

$$
\epsilon_{n x}=\frac{1}{m c} \sqrt{\left\langle x^{2}\right\rangle\left\langle P_{x}^{2}\right\rangle-\left\langle x P_{x}\right\rangle^{2}}
$$

Using Eqs. (9) and (10), the normalized emittance at the anode is calculated to be,

$$
\epsilon_{n x}=\kappa \sigma_{x}^{4} \frac{\alpha_{1}}{m c}
$$

where $\kappa$ is a constant that depends on the particle distribution function. For our particular case, Eqs. (10) and (11) need to be revisited since our laser spot size is an order of magnitude larger. The goal of the BNL gun is to have a longer charge lifetime, for which larger laser spot size is necessary. If the laser spot is large enough, for example $8.6 \mathrm{~mm}$ in our case compared to $0.35 \mathrm{~mm}$ in Ref. [30], operating the laser close to the edge of the cathode will introduce higher order nonlinearity from the transverse field. Therefore the calculation leading to Eq. (11) has to be expanded to include higher order nonlinearity coming from the transverse field. 

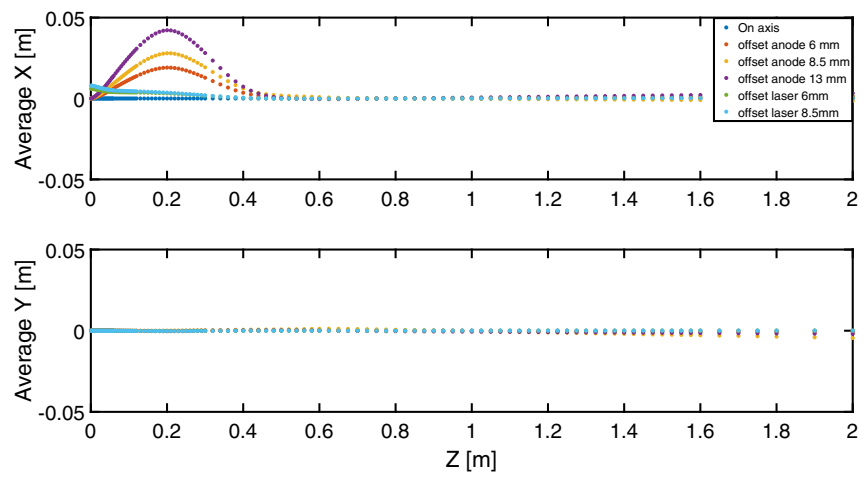

FIG. 10. Trajectory of the bunch center in the $\mathrm{X}$ and $\mathrm{Y}$ direction along the beam line.

The transverse nonlinearity in the electric field will introduce a higher order term in $\mathrm{P}_{x}$ such as,

$$
P_{x}=C x+\alpha_{1} x^{3}+\alpha_{2} x^{n}
$$

where $n$ is an integer such that $n>3$ and $\alpha_{2}$ is the coefficient of the external field nonlinear aberration. $\mathrm{P}_{x}$ could be written in terms of a multiple higher order terms as well. For simplicity, we considered the most dominant nonlinear term for this calculation. Using the same method of calculation as in [30], we obtain the following formulas for the square of the normalized emittance,

$$
\begin{aligned}
& \epsilon_{n x}^{2}=\frac{1}{m c}\left(\kappa_{1} \alpha_{1}^{2} \sigma_{x}^{8}+\kappa_{2} \alpha_{2}^{2} \sigma_{x}^{2(n+1)}\right) \quad \text { for } \mathrm{n}=\text { even } \\
& \epsilon_{n x}^{2}=\frac{1}{m c}\left(\kappa_{1} \alpha_{1}^{2} \sigma_{x}^{8}+\kappa_{2} \alpha_{2}^{2} \sigma_{x}^{2(n+1)}+2 \alpha_{1} \alpha_{2} \sigma_{x}^{(n+5)}\right) \\
& \quad \text { for } \mathrm{n}=\text { odd. }
\end{aligned}
$$

From the above equations it is clear that if the higher order nonlinear term is dominant, i.e., $\frac{\alpha_{1}}{\alpha_{2}} \ll 1$, the emittance growth will be dominated by the nonlinear term. If the nonlinearity leads to severe distortion of transverse phase space, it might not be feasible to fit $\mathrm{P}_{x}$ as a function of $x$. In the case of $8.5 \mathrm{~mm}$ laser offset in our simulation, fitting $x$ in terms of $\mathrm{P}_{x}$, we get,

$$
x=C P_{x}+\alpha_{1} P_{x}^{3}+\alpha_{2} P_{x}^{4}
$$

where the ratio of $\alpha_{1} / \alpha_{2}$ is of the order 1e-5. Therefore the $x^{4}$ term will dominate the emittance growth compared to the space charge related growth for $8.5 \mathrm{~mm}$ laser offset, which was seen from our simulation results. For the offset anode, the nonlinearity introduced at the exit of anode is minimal.

Figure 10 shows the $\mathrm{X}$ and $\mathrm{Y}$ trajectory of the center of the beam along the beam line for various anode offsets. Each point on the graph represents a "screen" at that point on the beam line. The maximum deviation on the $\mathrm{X}$ axis is at the center of the 1 st corrector magnet, which is expected.
TABLE IV. Beam size (100\% of the simulated ensemble) at the exit of anode $(5.6 \mathrm{~cm})$, at the center of $1 \mathrm{st} X$ corrector magnet $(20 \mathrm{~cm})$ and at the entrance of the solenoid $(60 \mathrm{~cm})$ downstream for various offset anode operations.

Anode

offset Diameter $(\mathrm{X} / \mathrm{Y}) \quad$ Diameter $(\mathrm{X} / \mathrm{Y}) \quad$ Diameter $(\mathrm{X} / \mathrm{Y})$

$\underline{(\mathrm{mm}) \quad \text { at } 5.6 \mathrm{~cm}(\mathrm{~mm}) \quad \text { at } 20 \mathrm{~cm}(\mathrm{~mm}) \quad \text { at } 60 \mathrm{~cm}(\mathrm{~mm})}$

\begin{tabular}{lrrr}
\hline 0 & $7.8 / 7.8$ & $14 / 14$ & $36 / 36$ \\
3 & $7.7 / 7.8$ & $14 / 14$ & $36 / 36$ \\
6 & $7.2 / 7.8$ & $14 / 14$ & $33 / 34$ \\
13 & $7 / 7.8$ & $12 / 14$ & $27 / 30$ \\
\hline \hline
\end{tabular}

The possibility of beam loss is maximum at the exit of anode (at $5.6 \mathrm{~cm}$ downstream), $20 \mathrm{~cm}$ downstream which is the center of the first $\mathrm{X}$ corrector and at the entrance of the solenoid $60 \mathrm{~cm}$ downstream. Beam diameters $(100 \%$ of the simulated ensemble) in the $\mathrm{X}$ and $\mathrm{Y}$ direction at these points are listed in Table IV. In all cases of offset-anode, the transverse kick in the dc gap was set such that the beam center passes through the center of the anode, albeit at an angle. The position of the 1st X corrector magnet was fixed due to mechanical constraint and $20 \mathrm{~cm}$ is the closest possible location for this magnet. Comparing the beam diameters at the exit of anode for all anode offsets, it is clear that there should not be any beam loss at the anode since the anode aperture is $3.6 \mathrm{~cm}$ in diameter. The beam size in all cases are more than 3 times smaller than the anode aperture. At $20 \mathrm{~cm}$ downstream, beam has enough clearance considering the $10 \mathrm{~cm}$ diameter beam pipe for all the cases except $13 \mathrm{~mm}$ deviation. For this particular case, beam is within $10 \mathrm{~mm}$ of the beam pipe wall and could potentially cause major beam loss. This restriction limited the maximum anode offset to $13 \mathrm{~mm}$ for this particular gun setup. Beam halo can be generated from betatron mismatch induced by the propagation of a space charge dominated beam in a focusing channel. The position, at which the shell particles start to separate from the core beam, can be evaluated by the quarter plasma wavelength of the beam [31,32]. For our particular situation, this position is $57 \mathrm{~cm}$ downstream from the cathode which is sufficiently far from the cathode. From Table IV, it is also evident that with increasing anode offset, the beam will experience a focusing in the $\mathrm{X}$ direction as it passes through the dc gap due to the transverse deflecting field. The mismatch in the sizes is partially recovered after the first solenoid. A quadrupole downstream could be used to match the beam sizes. The $\mathrm{X}$-Y emittance mismatch could be compensated by the proper positioning of a solenoid.

We note that transverse magnetic field in the dc gap will rotate the orientation of the spin polarization of the beam. The propagation of the beam in the dc gap is equivalent of beam propagating in a dipole magnetic field. We estimated the polarization orientation rotation for the maximum magnetic field, for $13 \mathrm{~mm}$ anode offset, and found it to 
be about 0.5 degrees. This rotation of the polarization vector can be corrected downstream with a Wien filter.

\section{CONCLUSION}

We proposed and showed simulation results for an offset anode scheme for dc polarized electron guns that can substantially increase charge lifetime from superlattice GaAs photocathodes. In this scheme, the effect of the high energy ion back bombardment is negated by essentially shifting these ions away from the cathode surface. This will permit operation with larger laser spot sizes while maintaining a constant fluence lifetime and resulting large charge lifetime. The improvement factor in charge lifetime can be ten or more depending on the overall size of the cathode, compared to the offset-laser scheme. The simulation results from Sec. III fully supports the claim of a higher charge lifetime using an offset anode scheme. In Sec. IV, we proved the feasibility of the scheme by performing detailed 3-D beam dynamics simulations and showing the complete transport of the beam through the gun with a negligible increase in the emittance. We have designed and fabricated necessary components, including an anode assembly capable of in-vacuum movement, to perform charge lifetime measurements with different anode offsets.

\section{ACKNOWLEDGMENTS}

The authors are grateful to Dr. Ferdinand Willeke, Dr. Jorg Kewisch from BNL, and Dr. Bruce Dunham from SLAC for useful discussions. The work was supported by U.S. Department of Energy under Contract No. DE-AC0298CH10886.

[1] R. Alley, H. Aoyagi, J. Clendenin, J. Frisch, C. Garden, E. Hoyt, R. Kirby, L. Klaisner, A. Kulikov, R. Miller et al., The Stanford linear accelerator polarized electron source, Nucl. Instrum. Methods Phys. Res., Sect. A 365, 1 (1995).

[2] G. Cates, V. Hughes, R. Michaels, H. Schaefer, T. Gay, M. Lubell, R. Wilson, G. Dodson, K. Dow, S. Kowalski et al., The bates polarized electron source, Nucl. Instrum. Methods Phys. Res., Sect. A 278, 293 (1989).

[3] W. Hartmann, D. Conrath, W. Gasteyer, H. Gessinger, W. Heil, H. Kessler, L. Koch, E. Reichert, H. Andresen, T. Kettner et al., A source of polarized electrons based on photoemission of gaasp, Nucl. Instrum. Methods Phys. Res., Sect. A 286, 1 (1990).

[4] C. K. Sinclair, P. A. Adderley, B. M. Dunham, J. C. Hansknecht, P. Hartmann, M. Poelker, J. S. Price, P. M. Rutt, W. J. Schneider, and M. Steigerwald, Development of a high average current polarized electron source with long cathode operational lifetime, Phys. Rev. ST Accel. Beams 10, 023501 (2007).
[5] T. Nishitani et al., Highly polarized electrons from gaasgaasp and ingaasalgaas strained-layer superlattice photocathodes, J. Appl. Phys. 97, 094907 (2005).

[6] C. Sinclair, The Working group M5 on Lepton-Hadron colliders, Snowmass 2001, Report No. SLAC-R-599, 2001.

[7] J. Grames, R. Suleiman, P. A. Adderley, J. Clark, J. Hansknecht, D. Machie, M. Poelker, and M. L. Stutzman, Charge and fluence lifetime measurements of a dc high voltage gaas photogun at high average current, Phys. Rev. Accel. Beams 14, 043501 (2011).

[8] K. Aulenbacher, Polarized beams for electron accelerators, Eur. Phys. J. Spec. Top. 198, 361 (2011).

[9] K. Aulenbacher, C. Nachtigall, H. Andresen, J. Bermuth, T. Dombo, P. Drescher, H. Euteneuer, H. Fischer, D. Harrach, P. Hartmann et al., The mami source of polarized electrons, Nucl. Instrum. Methods Phys. Res., Sect. A 391, 498 (1997).

[10] J. Grames, P. Adderley, J. Brittian, J. Clark, J. Hansknecht, D. Machie, M. Poelker, E. Pozdeyev, M. Stutzman, and K. Surles-Law, A biased anode to suppress ion backbombardment in a dc high voltage photoelectron gun, AIP Conf. Proc. 980, 110 (2008).

[11] J. Grames, P. Adderley, J. Brittian, D. Charles, J. Clark, J. Hansknecht, M. Poelker, M. Stutzman, and K. Surles-Law, Ion back-bombardment of gaas photocathodes inside dc high voltage electron guns, in Proceedings of the 21st Particle Accelerator Conference, Knoxville, TN, 2005 (IEEE, Piscataway, NJ, 2005), pp. 2875-2877.

[12] J. Clendenin, A. Brachmann, T. Galetto, D.-A. Luh, T. Maruyama, J. Sodja, and J. Turner, The slac polarized electron source, AIP Conf. Proc. 675, 1042 (2003).

[13] P. Adderley, J. Clark, J. Grames, J. Hansknecht, M. Poelker, M. Stutzman, R. Suleiman, K. Surles-Law, J. McCarter, and M. BastaniNejad, Cebaf $200 \mathrm{kv}$ inverted electron gun, in Proceedings of the 24th Particle Accelerator Conference, PAC-2011, New York, 2011 (IEEE, New York, 2011).

[14] G. Hoffstaetter, D. Trbojevic, and C. Mayes, CBETA design report, Technical Report No. BNL-114549-2017IR (Brookhaven National Laboratory (BNL), Upton, New York, 2017).

[15] V. Ptitsyn, Erl-ring and ring-ring designs for the erhic electron-ion collider, in North American Particle Accelerator Conf.(NAPAC'16) (2016), pp. 64-68, http:// accelconf.web.cern.ch/AccelConf/napac2016/papers/mob4 io02.pdf.

[16] O. Bruning and M. Klein, The large hardon electron collider, Mod. Phys. Lett. A 28, 1330011 (2013).

[17] D. Abbott, P. Adderley, A. Adeyemi, P. Aguilera, M. Ali, H. Areti, M. Baylac, J. Benesch, G. Bosson, B. Cade et al., Production of Highly Polarized Positrons Using Polarized Electrons at mev Energies, Phys. Rev. Lett. 116, 214801 (2016).

[18] E. Tsentalovich, Status of high intensity polarized electron gun project, Proc. Sci. PSTP2013 (2014) 043.

[19] H. Andresen, K. Aulenbacher, T. Dombo, P. Drescher, H. Euteneuer, H. Fischer, D. v. Harrach, P. Hartmann, P. Jennewein, K. Kaiser et al., operating experience with the mami polarized electron source, in Proceedings of 
the Workshop on Photocathodes for Polarized Electron Sources for Accelerators, Stanford (1993), pp. 2-12, http:// slac.stanford.edu/pubs/slacreports/reports10/slac-r-432.pdf.

[20] P. A. Adderley, J. Clark, J. Grames, J. Hansknecht, K. Surles-Law, D. Machie, M. Poelker, M. L. Stutzman, and R. Suleiman, Load-locked dc high voltage gaas photogun with an inverted-geometry ceramic insulator, Phys. Rev. Accel. Beams 13, 010101 (2010).

[21] B. Dunham, J. Barley, A. Bartnik, I. Bazarov, L. Cultrera, J. Dobbins, G. Hoffstaetter, B. Johnson, R. Kaplan, S. Karkare et al., Record high-average current from a highbrightness photoinjector, Appl. Phys. Lett. 102, 034105 (2013).

[22] E. Wang, High current polarized electron source for future erhic, AIP Conf. Proc. 1970, 050008 (2018).

[23] V. F. Software, Opera3d simulation software.

[24] S. Van Der Geer, O. Luiten, M. De Loos, G. Pöplau, and U. Van Rienen, 3d space-charge model for gpt simulations of high brightness electron bunches, in Institute of Physics Conference Series (2005), Vol. 175, p. 101, http:// inspirehep.net/record/1364299/files/ICAP02Proc .pdf\#page $=109$.

[25] W. Liu, S. Zhang, M. Stutzman, and M. Poelker, Effects of ion bombardment on bulk gaas photocathodes with different surface-cleavage planes, Phys. Rev. Accel. Beams 19, 103402 (2016).
[26] V. Shutthanandan, Z. Zhu, M. L. Stutzman, F. Hannon, C. Hernandez-Garcia, M. I. Nandasiri, S. V. Kuchibhatla, S. Thevuthasan, and W. P. Hess, Surface science analysis of gaas photocathodes following sustained electron beam delivery, Phys. Rev. Accel. Beams 15, 063501 (2012).

[27] M. Reiser, Theory and Design of Charged Particle Beams (John Wiley \& Sons, New York, 2008).

[28] Ionizer element for gpt.

[29] E. Pozdeyev, Ion trapping and cathode bombardment by trapped ions in dc photoguns, Phys. Rev. Accel. Beams 10, 083501 (2007).

[30] I. V. Bazarov, A. Kim, M. N. Lakshmanan, and J. M. Maxson, Comparison of dc and superconducting rf photoemission guns for high brightness high average current beam production, Phys. Rev. Accel. Beams 14, 072001 (2011).

[31] M. Reiser, P. O'Shea, R. Kishek, S. Bernal, P. Chin, S. Guharay, Y. Li, M. Venturini, J. Wang, V. Yun et al., The maryland electron ring for investigating space-charge dominated beams in a circular fodo system, in Proceedings of the 18th Particle Accelerator Conference, New York, 1999 (IEEE, New York, 1999), pp. 234-236.

[32] C. L. Bohnand and I. V. Sideris, Fluctuations Do Matter: Large Noise-Enhanced Halos in Charged-Particle Beams, Phys. Rev. Lett. 91, 264801 (2003). 